\title{
Gaussian beam with non-spiral optical vortex
}

\author{
Xiu-Min $\mathrm{Gao}^{1,2}$, Song $\mathrm{Hu}^{1}$, Jin-Song $\mathrm{Li}^{3}$, Han-Ming Guo ${ }^{2}$, Jian Wang ${ }^{1}$, Song-Lin Zhuang ${ }^{2}$ \\ ${ }^{1}$ Electronics and Information College, Hangzhou Dianzi University, Hangzhou, China; xiumin gao@yahoo.com.cn \\ ${ }^{2}$ Optics and Electronics College, University of shanghai for Science and Technology, Shanghai, China \\ ${ }^{3}$ Optics and Electronics College, China Jiliang University, Hangzhou, China
}

Received 24 November 2009; revised 28 December 2009; accepted 25 January 2010.

\begin{abstract}
Optical vortex has attracted much interest recently due to its novel properties and applications. In this paper, the focusing properties of Gaussian beam containing one non-spiral optical vortex are investigated by means of scalar diffraction theory. Simulation results show that topological charge of non-spiral optical vortex affects optical intensity distribution in focal region considerably, and non-spiral focal pattern may also occur. Multiple intensity peaks may appear companying with center main focal spot under condition of higher topological charge. In addition, the number of weak intensity peak outside of the center main intensity peak is related to the value of topological charge.
\end{abstract}

Keywords: Focusing Properties; Gaussian Beam; Optical Vortex; Scalar Diffraction Theory

\section{INTRODUCTION}

Singular optics studying optical vortex has grown rapidly recently because optical vortex has some interesting properties [1-5] and promising applications [6-8]. And common optical vortex refers the phase singularity in light wavefront. In practice, optical vortex contains optical orbital angular momentum, which can be used to construct highly versatile optical tweezers [9]. In the focal reign of focusing beam that contains optical vortex, the optical momentum can be transferred to micro-particle, namely, light energy can be transformed directly to dynamic energy of particle, which has been used to construct experimentally microscopic optical rotator. In recent years, there also has been much interest in optical vortex propagation [10-16]. From a fundamental perspective they enable the study of phase singularities in a dynamical context [11,12], where twists, loops and knots in the path of the vortices have been shown to appear [13-15].

Spiral optical vortex has attracted much and has been investigated intensively and extensively, however, nonspiral optical vortex induces little attention. In fact, non-spiral optical vortex may own more interesting properties due to more flexible vortex pattern. The present paper is aimed at studying focusing properties of Gaussian beam containing one non-spiral optical vortex are investigated by scalar diffraction theory. The principle of the focusing this non-spiral vortex Gaussian beam is given in Section 2. Section 3 shows the simulation results and discussions. The conclusions are summarized in Section 4.

\section{PRINCIPLE OF THE FOCUSING GAUSSIAN BEAM WITH ONE ASYMMET4IC OPTICAL VORTEX}

In the focusing system we investigated, the Gaussian beam contains one non-spiral optical vortex, and convergences through a lens. According to scalar diffractive theory, the relative amplitude distribution in focal region of the focusing optical system is [17],

$$
\begin{gathered}
U(r, \psi, z)=\frac{i}{\lambda} \int_{0}^{\alpha} \int_{0}^{2 \pi} E(\theta, \phi) \exp \left(i \varphi_{j}\right) \exp [-i k r \sin (\theta) \\
\cdot \cos (\phi-\psi)-i k z \cos (\theta)] \cdot \sin (\theta) d \theta d \phi
\end{gathered}
$$

where $E(\theta, \varphi)$ indicates the electric the amplitude of electric field of Gaussian beam that contains one non-spiral optical vortex, and can be written as,

$$
E(\theta, \phi)=A_{0} \cdot \exp \left[-\frac{\sin ^{2}(\theta)}{N A^{2} \cdot w^{2}}\right] \cdot \exp [i \pi \sin (m \phi)]
$$

where $A_{0}$ is a constant, where $w=w_{0} / r_{\mathrm{p}}, w_{0}$ is waist width (defined as radius) of the incident Gaussian beam.

Parameter $r_{\mathrm{p}}$ is the outer radius of the beam, and $m$ is the topological charge of the non-spiral optical vortex. The phase is the sine function of azimuthal angle, which is not spiral distribution and can be changed by topological charge. The optical intensity distribution in focal region can be calculated quantitatively by means of substituting Eq.2 into Eq.1. It should be note that 
the intensity distribution in focal region is proportional to the modulus square of Eq.1, and in this paper the modulus square of Eq.1 is calculated numerically.

\section{RESULTS AND DISCUSSIONS}

Without losing generality and validity, the intensity is normalized by optical intensity maximum, and it was proposed that $N A=0.6, w=1$. Firstly, the topological charge of the optical vortex is chosen as 1. Figure 1(a) illustrates the phase wavefront distribution under condition of $m=1$ in polar coordinates. The radial coordinate indicates the phase value. It can be seen that the optical vortex is non-spiral and turns on off-axis distribution.

The Figure 1(b) shows that optical intensity in focal region of this kind of vortex Gaussian beam. It should be noted that $V r$ and $\Psi$ indicate radial and azimuthal coordinates, respectively. $\Psi$ ranges from 0 to $2 \pi$. And the distance unit in radial direction is $k^{-1}$, here $k$ is the wave number of the focusing beam. We can see that the focal spot is asymmetric remarkably, and does not locate at optical axis. The cause of this focal pattern is the asymmetry characteristics of the optical vortex that was embedded in the incident Gaussian beam. There the symmetry property of the optical vortex affects focal pattern.

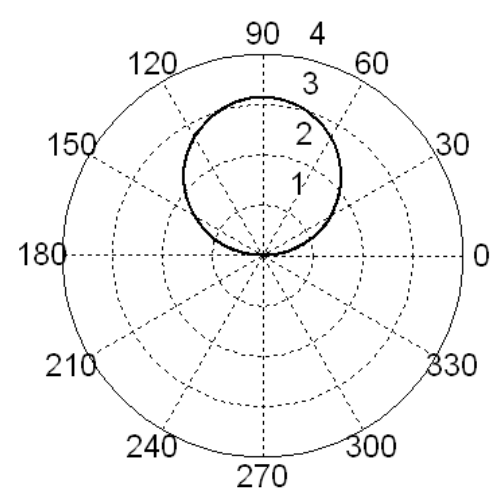

(a)

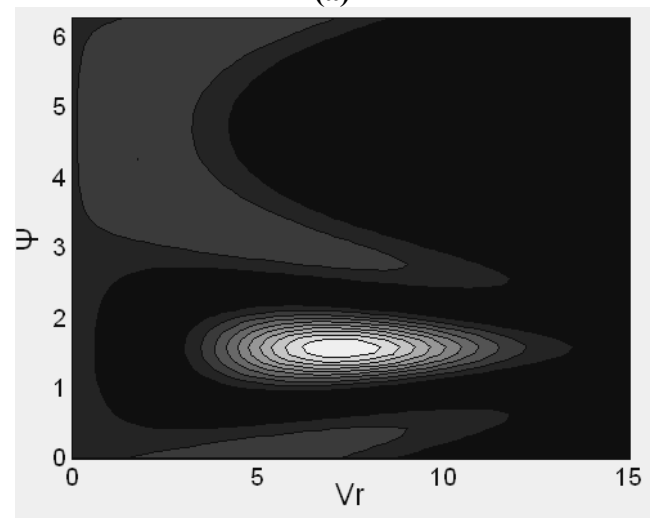

(b)

Figure 1. The (a) phase distribution for $m=1$ and the (b) corresponding focal intensity distribution.
Topological charge is important parameter optical vortex, here, the effect of different topological charge on focal pattern is investigated. Figure 2(a) illustrates the phase distribution for $m=2$. It can be seen that there are four phase maximums and locate around optical axis. Topological charge $\mathrm{m}$ affects phase wavefront distribution considerably. Figure 2(b) show us the intensity distribution in focal region under condition of $m=2$.

From this figure, we can see that there is one center main intensity spot on optical axis. Four optical intensity peaks come into being outside of center main intensity peak. And the center main intensity peaks also extends outside to form four intensity branches. Therefore, there are four local intensity minimums appear between center main peak and outside four subordinate intensity peaks. This phenomenon is very interesting and can be used to restrict those micro particles whose refractive index is lower than that of the surrounding medium, and this kind of condition is common in life science. In addition, the whole focal pattern may also be employed to construct novel optical trap. By comparing Figure 2 with Figure 1, the optical intensity distribution in focal region of the Gaussian beam containing one non-spiral optical vortex can be altered remarkably by the topological charge.

In order to get insight into the effect of topological change more deeply, focusing properties for other dif-

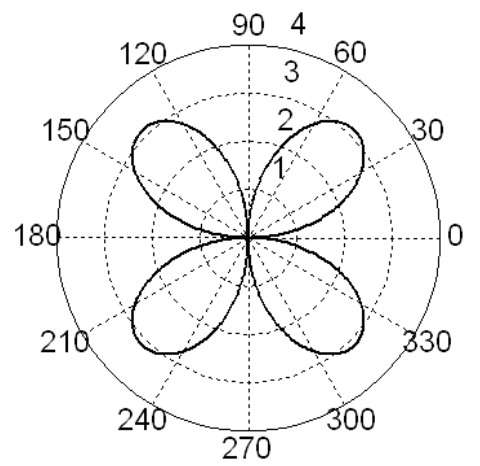

(a)

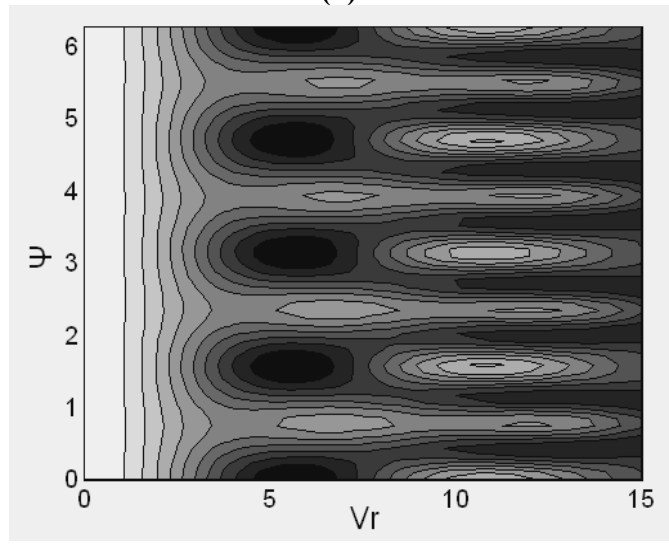

(b)

Figure 2. The (a) phase distribution for $m=2$ and the (b) corresponding focal intensity distribution. 
ferent $\mathrm{m}$ are also investigated. Figure 3 shows the phase distribution for $m=3$ and the corresponding focal intensity distribution. There are three phase maximums and turns symmetric distribution around optical axis. From Figure 3(b), it can be seen that for this case, the focal pattern contains one center main intensity peak with three relative weak intensity peaks outside. And there are no local intensity minimums between center peak and outside three peaks. Number of subordinate intensity peaks is related to the number of topological charge $m$. Simulation shows that number of subordinate intensity peaks equals $m$ when $m$ is odd integral number.

We also studied effect of some other value of topological charge on focal pattern. Figure 4 illustrates phase distribution for $m=4$ and the corresponding focal intensity distribution. It can be seen that there are eight phase maximums, and also eight weak intensity peaks around center main intensity peak in focal region, as shown in Figure 4(b). The number of subordinate intensity peaks is two times of the parameter $m$ when $m$ is even integral number.

From all above focal pattern evolution on increasing topological charge, we can seen that topological charge of non-spiral optical vortex affects optical intensity distribution in focal region very obviously, and non-spiral focal pattern may also occur. Multiple intensity peaks

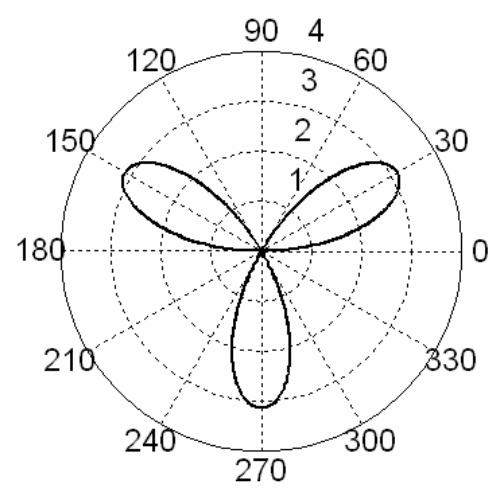

(a)

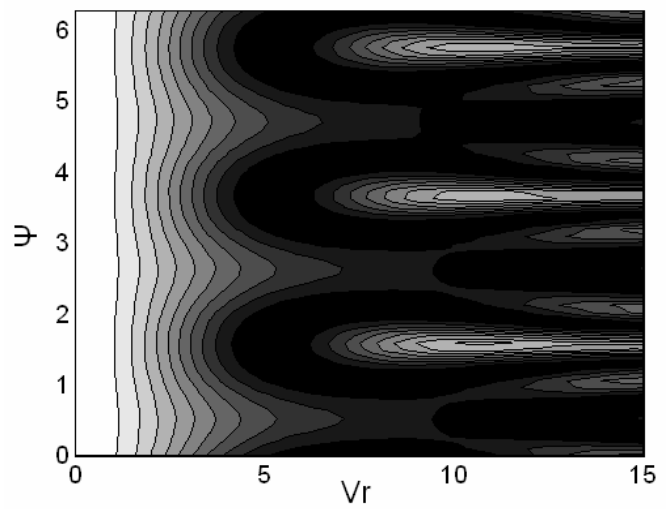

(b)

Figure 3. The (a) phase distribution for $m=3$ and the (b) corresponding focal intensity distribution. may appear companying with center main focal spot under condition of higher topological charge. In addition, the number of weak intensity peak outside of the center main intensity peak is related to the value of topological charge. When topological charge is odd number, the number of weak intensity peak equals the value of topological charge, while, the number of weak intensity peak is two times the value of topological charge if topological charge is even number. In optical trapping system, it is usually deemed that the forces exerted on the particles in light field include two kinds of forces, one is the gradient force, which is proportional to the intensity gradient; the other is the scattering force, which is proportional to the optical intensity [18]. Therefore, the tunable

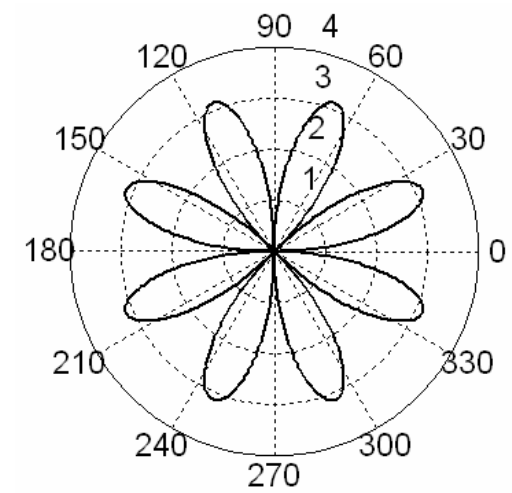

(a)

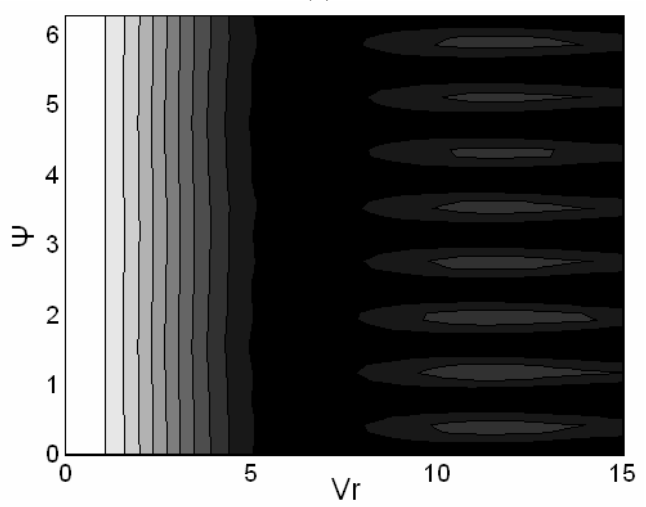

(b)

Figure 4. The (a) phase distribution for $m=4$ and the (b) corresponding focal intensity distribution.

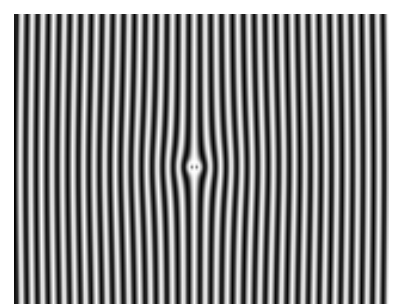

(a)

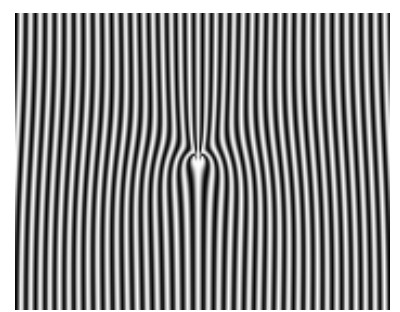

(b)
Figure 5. Holograms for generation this non-spiral optical vortex for (a) $m=1$ and (b) $m=2$, respectively. 
focal intensity distribution predicts that the focusing properties of this kind of beam can be employed to construct controllable trap.

We are now going on investigating the focusing properties of this kind of vortex beam. Next step, the attention will be focused on the experimental research and extends study into vector optical domain. Figure 5 illustrates holograms for generating this non-spiral optical vortex, which may be employed in our future experiment. These holograms can be obtained conveniently by calculating numerically optical interference diagram.

\section{CONCLUSIONS}

The focusing properties of Gaussian beam containing one non-spiral optical vortex are investigated by scalar diffraction theory in this paper. Calculation results show that topological charge of non-spiral optical vortex affects optical intensity distribution in focal region remarkably, and asymmetric focal pattern may also occur for lower topological charge. Multiple intensity peaks may come into being companying with center main focal spot under condition of higher topological charge. In addition, the number of weak intensity peak equals the value of topological charge under condition of odd number topological charge, while, the number of weak intensity peak is twice times the number of topological charge for even number topological charge.

\section{ACKNOWLEDGMENT}

This work was supported by National Basic Research Program of China (2005CB724304), National Natural Science Foundation of China (60708002, 60878024, 60778022, 60807007), China Postdoctoral Science Foundation (20080430086), Shanghai Postdoctoral Science Foundation of China (08R214141), and Shanghai Leading Academic Discipline Project (S30502).

\section{REFERENCES}

[1] Thomas, V.D. and Taco, D.V. (2009) Evolution of singularities in a partially coherent vortex beam. Optical Society of America B, 26, 741-744.

[2] Coullet, P., Gil, L. and Rocca, F. (1989) Optical vortices. Optics Communications, 73, 403-408.

[3] Palacios, D.M., Maleev, I.D., Marathay, A.S. and Swartzlander, G.A. (2004) Spatial correlation singularity of a vortex field. Physical Review Letters, 92, 143905-4.
[4] Rozas, D.Z., Sacks, S. and Swartzlander, G.A. (1997) Experimental observation of fluidlike motion of optical vortices. Physical Review Letters, 79, 3399-3402.

[5] Filippus, S.R. (2004) Distribution of angular momentum and vortex morphology in optical beams. Optics Communications, 242, 45-55.

[6] Lee, W.M., Yuan, X.C. and Cheong, W.C. (2004) Optical vortex beam shaping by use of highly efficient irregular spiral phase plates for optical micromanipulation. Optics Letters, 29, 1796-1798.

[7] Ladavac, K. and Grier, D.G. (2004) Microoptomechanical pumps assembled and driven by holographic optical vortex arrays. Optics Express, 12, 1144-1149.

[8] Cojoc, D., Garbin, V., Ferrari, E., Businaro, L., Romamato, F. and Fabrizio, E.D. (2005) Laser trapping and micro-manipulation using optical vortices. Microelectronic Engineering, 77-78, 125-131.

[9] Vladlen, G., Shvedov, A.S., Desyatnikov, A.V., Krolikowski, R.W. and Kivshar, Y.S. (2009) Optical guiding of absorbing nanoclusters in air. Optics Express, 17, 5743-5757.

[10] Soskin, M.S. and Vasnetsov, M.V. Singular Optics, in Progress in Optics 42, Wolf, E. Ed. (Elsevier 2001).

[11] Indebetow, G. (1993) Optical vortices and their propagation. Journal of Modern Optics, 40, 73-87.

[12] Rozas, D., law, C.T. and Swartzlander, G.A. (1997) Propagation dynamics of optical vortices. Journal of the Optical Society of America B, 14, 3054-3065.

[13] Berry, M.V. and Dennis, M.R. (2001) Knotted and linked phase singularities in monochromatic waves. Proceedings of the Royal Society A, 457, 2251-2263.

[14] Berry, M.V. and Dennis, M.R. (2001) Knotting and unknotting of phase singularitites: Helmholtz waves, paraxialwaves and waves in $2+1$ spacetime. Journal of Physical A, 34, 8877-8888.

[15] Leach, J., Dennis, M., Courtial, R.J. and Padgett, M.J., (2005) Vortex knots in light. New Journal of Physics, 7, $1-11$.

[16] Baumann, S.M., Kalb, D.M., MacMillan, L.H. and Galvez, E.J. (2009) Propagation dynamics of optical vortices due to Gouy phase. Optics Express, 17, 98189827.

[17] Gu, M. (2000) Advanced Optical Imaging Theory (Springer, Heidelberg)

[18] Visscher, K. and Brakenhoff, G.J. (1992) Theoretical study of optically induced forces on spherical particles in a single beam trap I: Rayleigh scatterers. Optik, 89 174-180. 